\title{
The Development and Structure of Cornalean Flowers and Fruits.
}

\author{
Brian A. Atkinson ${ }^{1}$, Teresa Sawyer ${ }^{2}$, Gar W. Rothwell ${ }^{1,3}$, and Ruth A. Stockey ${ }^{1}$ \\ 1. Department of Botany and Plant Pathology, Oregon State University, Corvallis, Oregon, USA. \\ 2. Electron Microscope Facility, Oregon State University, Corvallis, Oregon, USA. \\ 3. Department of Environmental and Plant Biology, Ohio University, Athens, Ohio, USA.
}

The order Cornales (dogwood order) is the earliest diverging lineage within the most diverse group of flowering plants the asterids, which number over 80,000 living species [1]. This order contains an impressive diversity of flower and fruit morphologies, which makes Cornales an important resource for understanding the early evolutionary diversification of asterids. Fortunately, the Cornales is exceptional in having an excellent fossil record dating back to the Late Cretaceous (100-66 Ma), thus providing a rich source of crucial data for a wide range of evolutionary studies [2, 3]. Recent investigations have generated several hypotheses of cornalean evolution and systematics that remain untested by comparative morphological and anatomical studies, and fossils provide critical data both for testing those hypotheses and for reconstructing the early evolutionary history of the group [3].

Due to the systematic importance of the Cornales, the paleobotany lab at Oregon State University has begun an in-depth initiative to study the early evolution of this order. Paleobotancial studies of Cornales have already begun in which Late Cretaceous cornalean flowers and fruits are being characterized. However, in order to develop a broader understanding of cornalean reproductive biology, it is essential to study flowers and fruits of living representatives.

Preliminary results from fossil analyses suggest that a number of Late Cretaceous species may be closely related to Nyssa (Nyssaceae) and Davidia (Davidiaceae). Therefore, it is necessary to analyze developing flowers and fruits of Nyssa sylvatica and Davidia involucrata. The aim of this study is to characterize the morphology and anatomy of young and mature flowers and fruits, and to relate them to comparable features of the fossil species. The overarching goal is to reveal developmental processes and patterns that lead to significant evolutionary changes in fruit structure.

Samples are taken from Nyssa and Davidia trees that are cultivated on the Oregon State University campus. During November 2014, mature fruits Nyssa and Davidia were collected and sectioned transversely and longitudinally before chemical fixation. Mature fruits were fixed in Formalin - Acetic Acid - Alcohol (FAA) for several days. Fruits were then dehydrated in an ethanol series (30\% for two days, $50 \%$ for three days, $70 \%$ for four days, and $95 \%$ for four days). After dehydration the specimens infiltrated and embedded with ethanol-glycol methacrylate (Technovit 7100 embedding kit, Electron Microscopy Sciences, Hatfield, Pennsylvania, USA) plastic, at sequential ratios: 2:1, 1:1, and 2:1. Then specimens were fully embedded in the plastic. Fruits were sectioned using an AO 820 rotary microtome at a thickness of 5 microns. Microtome sections were stained with toluidine blue ( $\mathrm{pH} 4.2)$ and mounted on microscope slides with mounting medium. During the spring mature flowers will be collected and prepared using the same steps as above. Prepared specimens will be photographed using a BetterLight digital scanning camera (Better Light, Placerville, California, USA).

Samples collected for scanning electron microscopy were chemically fixed with a diluted Karnovsky solution for 60 hours at $4^{0} \mathrm{C}$. Flowers were dehydrated in an ethanol serial solution for critical point 
drying. Images were acquired using a Quanta 600 FEG, FEI, Hillsboro, OR, USA.

The results from this study will provide important data regarding the morphology, anatomy, and development of cornalean flowers and fruits. We hypothesize that there are different developmental processes and patterns of developmental change that lead to mature structural differences between Nyssa and Davidia. These studies will provide a test of this hypothesis. Furthermore, the data yielded from this study will provide a framework for working with fossil flowers and fruits that represent different stages of development. Using a multidisciplinary approach consisting of developmental and paleobotanical research we have an opportunity to shed light on the evolutionary processes that have shaped the evolution and diversity of reproductive organs within Cornales [4].

References:

[1] APG, Botanical Journal of the Linnean Society 161 (2009), p.105.

[2] C Fan and QY, Xiang American Journal of Botany 90 (2003), p. 1357.

[3] QY Xiang et al, Molecular Phylogenetics and Evolution 59 (2011), p. 123.

[4] The authors acknowledge funding from the National Science Foundation, grant number DGE1314109. 